\title{
Dynamic Analysis of Coupled Sawtooth and Rectangle Cellular Neural Networks with Application in HBV Patients' B-Scan Images
}

\author{
Mian Wang ${ }^{a} \quad$ Lequan Min $^{a} \quad$ Gerhard Litscher ${ }^{a}$ b $\quad$ Min Li ${ }^{a}$ \\ aSchool of Mathematics and Physics, University of Science and Technology Beijing, Beijing, \\ PR China; ${ }^{\mathrm{b}}$ Research Unit of Biomedical Engineering in Anesthesia and Intensive Care \\ Medicine and TCM Research Center Graz, Medical University of Graz, Graz, Austria
}

\section{Keywords}

Cellular neural network · Sawtooth and rectangle patterns · Grayscale image · Global task .

Local rule · Liver image

\begin{abstract}
Liver damage caused by hepatitis B virus (HBV) infections is diffuse. Live medical images and FibroScan images show that it is inhomogeneous. Modeling and interpreting such phenomena are both important for theoretical research and practical application. The cellular neural networks (CNNs) introduced by Chua and Yang can model widely observed patterns in both nonbiological and biological media. The sawtooth and rectangle (SR) CNN introduced by Chua and colleagues is able to generate SR-shaped patterns for any random-input grayscale patterns, which are also similar to some chronic HBV-infected patients' liver B-scan images. This paper will mathematically study the dynamic behaviors of the SR CNN, numerically simulate the output images of the SR CNN, and biologically interpret the relationships between the output images of the SR CNN and the patterns in HBV-infected patients' liver B-scan images. Our research results show that the SR CNN may be used as a candidate for modeling liver infections.

\section{Introduction}

Hepatitis B is one of the serious infectious diseases which threaten global human health. Two billion people have been infected with the hepatitis B virus (HBV), and no less than 240 million persons are chronically infected with HBV [1]. HBV-caused chronic infection can lead to the later development of cirrhosis of the liver or liver cancer [1]. Most chronic HBV patients 
need long-term therapy. After a patient's plasma HBV load has been reduced below the lower limit of detection, recovery from liver damage (assessed via medical imaging) becomes important for evaluating and predicting the efficacy of treatment. Liver medical and FibroScan images show that liver damage in chronically HBV-infected patients is inhomogeneous. The interpretation of medical images of chronic HBV patients' livers is important. To our knowledge, no models have up to now been presented to describe chronic HBV patients' liver damage.

The cellular neural network (CNN) introduced by Chua and Yang [2, 3] is a new information processing paradigm. The standard CNN consists of locally coupled matrix differential equations. Such locally coupled systems may be suitable to model biological systems consisting of homogeneous media. Chua stated that complex patterns and structures, which are widely observed in both inanimate (nonbiological) and biological media, can be modeled and studied via the CNN paradigm in an in-depth and unified way [4]. Now, the CNN and its generalization have been widely studied for practical application in image and video signal processing, robotic biological vision, higher brain functions, and cryptography [5-18].

In his monograph on the CNN [5], Chua invested spontaneous pattern formations with several kinds of coupled CNNs from random initial conditions. Thiran et al. [19] provided a categorization of patterns that can be formed with $3 \times 3$ template. They found that for the $\mathrm{CNN}$ with a "square cross template"

$$
A=\left[\begin{array}{lll}
0 & c & 0 \\
c & a & c \\
0 & c & 0
\end{array}\right], \quad B=\left[\begin{array}{lll}
0 & 0 & 0 \\
0 & 0 & 0 \\
0 & 0 & 0
\end{array}\right], \quad Z=0,
$$

if the template parameters satisfy the following inequalities

$$
\begin{aligned}
& a+2 c-1>0, \\
& a-1<0, \\
& c>0,
\end{aligned}
$$

any initial random-state input pattern will give rise to a stable output pattern in which sawtooth and rectangle (SR) shapes coexist, which have some characteristics similar to those found in HBV-infected patients' liver B-scan images. Now, we present 3 arising problems as follows:

- Why is the CNN with the template whose parameters satisfy Inequations (2)-(4) able to generate SR-shaped patterns?

- How does one define the SR-shaped patterns exactly?

- Is there some biological signification of the functions of the SR CNN?

First, this paper presents 7 definitions which describe some fundamental characteristics of the output patterns of the SR CNN. We will indicate the biological indications of these definitions. Second, we show that the template parameters of the square cross template CNN (named SR CNN) should satisfy Inequations (2)-(4). Third, the study establishes a set of theorems to show the properties of output patterns of the SR CNN. Finally, we simulate the dynamics of some SR CNNs and then compare some output patterns of the SR CNN with some people's digital liver B-scan images. The results suggest that the dynamic behaviors of some SR CNNs may provide a possible way of interpretation of HBV-infected patients' digital liver B-scan images.

The rest of this paper is organized as follows. The section entitled Sawtooth and Rectangle CNN introduces some definitions related to the behaviors of the SR CNN and describes the global task and the local rules of the SR CNN. The section entitled Mathematical Analysis establishes a group of theorems to determine the properties of the output patterns of the SR CNN. Numerical simulation examples for comparison with several HBV-infected patients' liver images are given in the next section. The final section states some conclusions. The proofs of the postulated theorems are presented in the Appendix. 


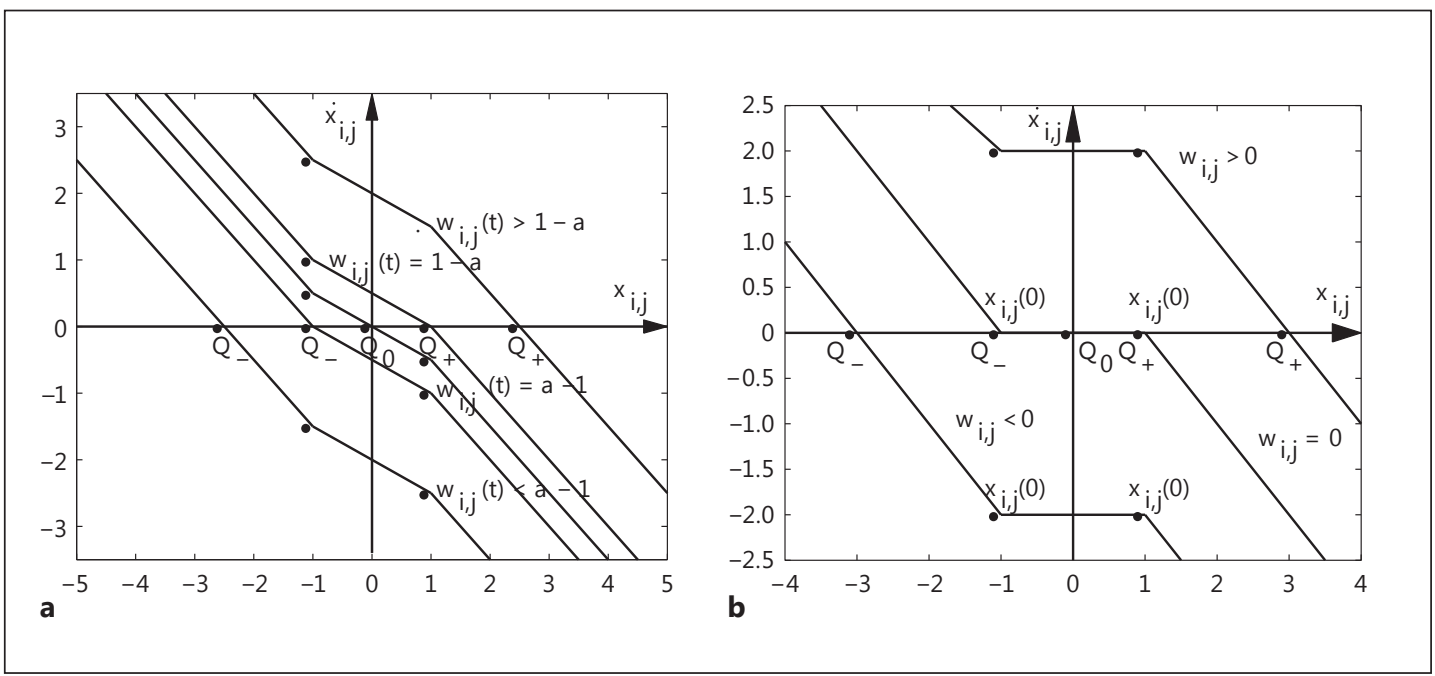

Fig. 1. The dynamic routes of the SR CNN with $\mathrm{a}<1$ (a) and $\mathrm{a}=1$ (b).

\section{Sawtooth and Rectangle CNN}

\section{Standard CNN Equation and SR CNN}

The standard CNN with a $3 \times 3$ template is composed of a 2-dimensional $M \times N$ array of cells. Each cell is denoted by $C_{i, j}$, where $i=1,2, \ldots, M$ and $j=1,2, \ldots, N$. The dynamics of $C_{i, j}$ is given by Chua [5]:

$$
\dot{x}_{i, j}=-x_{i, j}+\sum_{k=-1}^{1} \sum_{l=-1}^{1} a_{k, l} y_{i+k, j+l}+\sum_{k=-1}^{1} \sum_{l=-1}^{1} b_{k, l} u_{i+k, j+l}+z,
$$

where $u_{i, j}, x_{i, j}$, and $y_{i, j}$ are the input, state, and output variables of the cell, and $a_{k, l}{ }^{\prime} \mathrm{s}, b_{k, l}{ }^{\prime} \mathrm{s}$, and $z$ are the elements of the A template, the B template, and the threshold, respectively. The output $y_{i, j}$ is the piecewise linear function given by

$$
y_{i+k, j+l}=1 / 2\left(\left|x_{i+k, j+l}+1\right|-\left|x_{i+k, j+l}-1\right|\right) .
$$

The state equation of the SR CNN has the form

$$
\begin{aligned}
\dot{x}_{i, j}(t) & =-x_{i, j}(t)+a y_{i, j}(t)+c\left(y_{i-1, j}(t)+y_{i, j-1}(t)+y_{i, j+1}(t)+y_{i+1, j}(t)\right) \\
& =-x_{i, j}(t)+a y_{i, j}(t)+w_{i, j}(t) .
\end{aligned}
$$

The dynamic routes of the SR CNN are shown in Figures 1 and 2. Since the translation term $w_{i, j}$ is dependent on time $t$, this makes the dynamic routes of the SR CNN very complex.

\section{Some Definitions Related to SR CNN}

In order to describe possible output images (stable equilibria) of the SR CNN, we introduce the following definitions [similar to 19]:

Definition 1. Let $U=\left(u_{i, j}\right)_{M \times N}$ be a grayscale image with $M \times N$ pixels. A pixel $C_{i, j}$ at the $(i, j)$ th position with grayscale $u_{i, j}$ is said to be inherent active if $y_{i, j}(t) \equiv 1$. A pixel $C_{i, j}$ is said to be inherent passive if $y_{i, j}(t) \equiv-1$.

REMARK 1. Inherent active pixels can be interpreted as normal liver cells. Inherent passive pixels can be interpreted as HBV-infected or damaged liver cells.

Definition 2. A pixel $C_{i, j}$ is said to be inherent neutral if $y_{i, j}(t) \in(-1,1)$. 


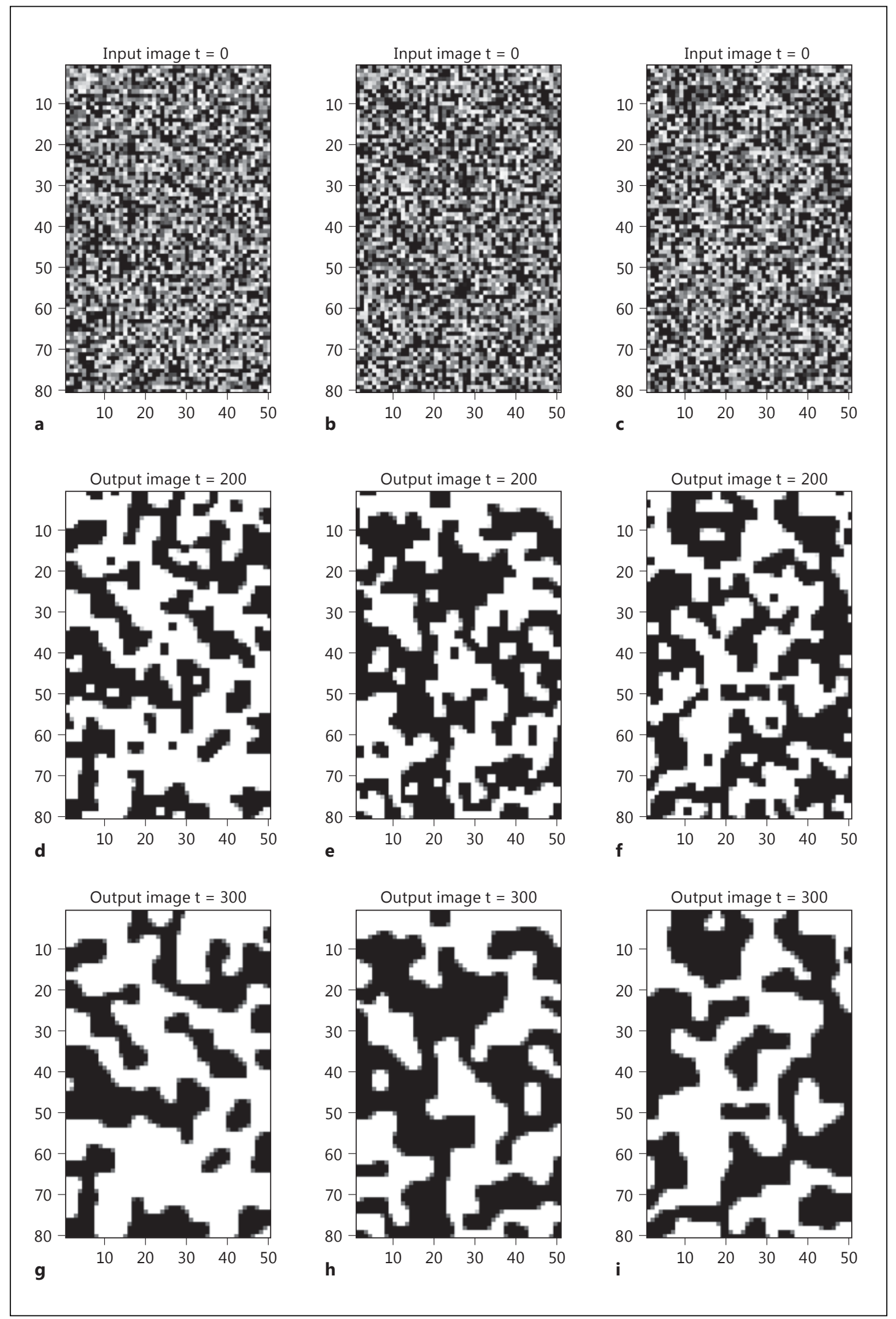

Fig. 2. Inputs and outputs of 3 simulation examples. a-c Random input images. $\mathbf{d}-\mathbf{i}$ Corresponding output images. a-f Template parameter $a=1$. $\mathbf{g}-\mathbf{i}$ Template parameter $a=0.97$. 
REMARK 2. Inherent neutral pixels can be interpreted as being uncertain (infected or recovered) liver cells.

DEFINITION 3. For an SR CNN, let $U=\left(u_{i, j}\right)_{M \times N}$ be a grayscale image with $M \times N$ pixels. A pixel $C_{i, j}$ at the $(i, j)$ th position with grayscale $u_{i, j}$ is said to be final active if there is a constant $t^{*}>0$ such that $y_{i, j}(t) \equiv 1, \forall t>t^{*}$. A pixel is said to be final passive if there is a constant $t^{*}>0$ such that $y_{i, j}(t) \equiv-1, \forall t>t^{*}$. A pixel $C_{i, j}$ is said to be final neutral if there is a constant $t^{*}>0$ such that $y_{i, j}(t) \in(-1,1), \forall t>t^{*}$.

REMARK 3. Final active pixels can be interpreted as liver cells finally becoming normal. Final passive pixels can be explained as liver cells finally becoming HBV infected or damaged. Final neutral pixels can be interpreted as liver cells finally becoming uncertain (infected or recovered).

DEFINITION 4. For an SR CNN, let $U=\left(u_{i, j}\right)_{M \times N}$ be a grayscale image with $M \times N$ pixels. An active network is the set consisting of connected inherent active cells and connected final active cells. A passive network is the set consisting of connected inherent passive cells and connected final passive cells. A neutral network is the set consisting of connected inherent neutral cells and connected final neutral cells.

REMARK 4. An active (passive) network can be interpreted as some connected liver cells which will finally become normal (infected). Final neutral pixels can be interpreted as liver cells finally becoming uncertain (infected or recovered).

DEFINITION 5. A sawtooth black/white island is a sawtooth-shaped area which is composed of edge-connected black/white pixels, and every black/white pixel has at least 3 black/white neighbor pixels, 2 of them connected in a square cross way.

REMARK 5. For some initial grayscale input images, the stable output images of the SR CNN can generate sawtooth black/white islands, which can be interpreted as sawtooth-shaped normal/HBV-infected cells.

DEFINITION 6. A rectangle black/white island is a rectangle-shaped area which is composed of edge-connected black/white pixels, and every black/white pixel has at least 2 black/white neighbor pixels connected in a square cross way.

REMARK 6. For some initial grayscale input images, the stable output images of the SR CNN can generate rectangle black/white islands, which can be interpreted as rectangle-shaped normal/HBV-infected cluster cells.

REMARK 7. The output of an active network is a black (grayscale is +1 ) pattern. The output of a passive network is a white (grayscale is -1 ) pattern. The output of a neutral network is a gray (grayscale is in $[-1,1]$ ) pattern.

\section{Global Tasks and Local Rules for the SR CNN}

The dynamic properties of a CNN are described via its global tasks and local rules [5]. Now, we propose the global tasks and local rules for the SR CNN.

\section{Global Task of the Binary Cow Patch CNN}

1. Given: a static binary image $U$.

2. Initial state: $X(0)=U$.

3. Boundary conditions: state variable is reflected across the boundary.

4. Output: $Y(t) \rightarrow Y(\infty)$ consists of sawtooth patterns and rectangle patterns.

\section{Local Rules of the Binary Cow Patch CNN $u_{i, j} \quad y_{i, j}(\infty)$. \\ 1. White/black $\rightarrow$ black if $C_{i, j}$ is inherent active or final active. \\ 2. White/black $\rightarrow$ white if $C_{i, j}$ is inherent passive or final passive. \\ 3. White/black $\rightarrow$ gray if $C_{i, j}$ is inherent neutral or final neutral.}




\section{Mathematical Analysis}

In the following discussion, we present several theorems on the properties of the output images processed by the SR CNN.

THEOREM 1. Let the positions of CNN template parameters be described by Matrix (1). Assume that a $\leq 1$; if a CNN can generate gray sawtooth-shaped and gray rectangle-shaped patterns from a random initial pattern, then the following parameter inequalities must hold:

$$
\begin{aligned}
& a+2 c-1 \geq 0, \\
& a-1 \leq 0, \\
& c>0 .
\end{aligned}
$$

THEOREM 2. A black pixel $C_{i, j}$ is inherent active if there are at least 3 fixed black neighbors on its nearest square cross lines.

THEOREM 3. A white pixel $C_{i, j}$ is inherent passive if there are at least 3 fixed white neighbors on its nearest square cross lines.

THEOREM 4. Let a pixel $C_{i, j}$ be neither inherent active nor inherent passive. If there is a constant $t^{*}>0$ such that $\forall t \geq t^{*}$,

1. $w_{i, j}(t) \geq 1-a$ if $a<1$ or $w_{i, j}(t)>0$ if $a=1$, then $y_{i, j}(\infty)=1$,

2. $w_{i, j}(t) \leq a-1$ if $a<1$ or $w_{i, j}(t)<0$ if $a=1$, then $y_{i, j}(\infty)=-1$,

3. $a-1<w_{i, j}(t)<1-a$ if $a<1$ or $w_{i, j}(t)=0$ if $a=0$, then $y_{i, j}(\infty)=x_{i, j}\left(t^{*}\right)$.

THEOREM 5. For any random initial input image, the stable output image of the SR CNN has the following properties:

1. There is no isolated gray pixel surrounded by black or white pixels.

2. There are no 2 isolated edge-connected gray pixels surrounded by black or white pixels.

COROLLARY 1. Horizontal lines or vertical lines consisting of 1 connecting pixel cannot exist in stable output patterns generated by the SR CNN.

THEOREM 6. For any random initial input image, the stable output image of the SR CNN with $a=1$ can output unchanged sawtooth islands or unchanged rectangle islands.

\section{Numerical Simulation}

In this section, we will first provide 3 experimental simulation examples in 3 initial states for designing the SR CNN.

Now let us consider the following SR CNN template for simulation:

$$
A=\left[\begin{array}{ccc}
0 & 0.2 & 0 \\
0.2 & a & 0.2 \\
0 & 0.2 & 0
\end{array}\right], B=\left[\begin{array}{lll}
0 & 0 & 0 \\
0 & 0 & 0 \\
0 & 0 & 0
\end{array}\right], Z=0,
$$

Figure $2 \mathrm{a}-\mathrm{c}$ shows random images with $40 \times 60$ pixels generated via the MATLAB command $2(\operatorname{rand}(50,80)-0.5)$. Figure $2 \mathrm{~d}-\mathrm{f}$ shows the corresponding output images generated via the SR CNN with Template (10) where $a=1$. Figure $2 \mathrm{~g}$ and h shows the corresponding output images generated via the SR CNN with Template (10) where $a=0.97$. The connected regions in these images are larger than the ones shown in Figure $2 d-f$. Observe that although there are 3 different random images as inputs, the output images show similar SR-like patterns.

Figure 3a-f displays the digital liver tissue B-scan images of 6 individuals, which were scanned with an EUB-8500 Ultrasound Scanner. We will see that the SR CNN may simulate these images. Figure $3 a$ is the digital liver tissue B-scan image of a healthy person who has a 


\section{Integrative \\ Medicine}

International
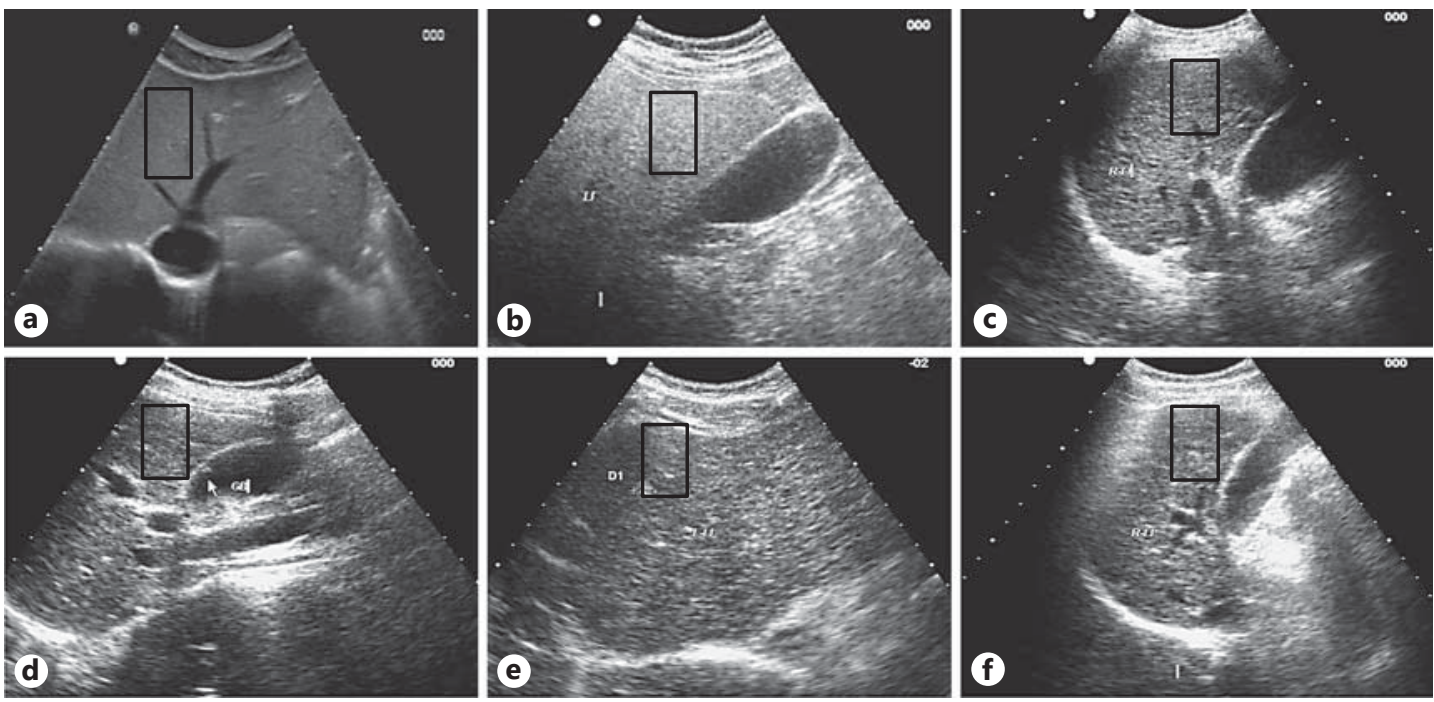

Fig. 3. Digital liver B-scan images. a Normal person. b Healthy hepatitis B virus (HBV) carrier. c-f Four chronic HBV patients.

healthy liver. Figure $3 \mathrm{~b}$ is the digital liver tissue B-scan image of a patient who is infected with HBV but has normal ALT levels and fatty liver. The other parts of Figure 3 show the digital liver tissue B-scan images of 4 chronic HBV patients. The clinical diagnoses for the 4 patients are listed as follows:

1. The liver tissue shown in Figure $3 \mathrm{c}$ has some liver damage but no fibrosis.

2. The liver tissue shown in Figure $3 \mathrm{~d}$ has mild fibrosis.

3. The liver tissue shown in Figure 3e has mild cirrhosis.

4. The liver tissue shown in Figure $3 \mathrm{f}$ has been diagnosed as developing towards mild cirrhosis.

Figure $4 \mathrm{a}-\mathrm{f}$ displays the marked insets (each $80 \times 50$ pixels in size) from the respective images shown in Figure 3a-f. Liver nonuniformity is an important index for evaluating liver damage. We take the mean gray value (mean $1 ; 90.24$ ) \pm standard deviation (standard $1 ; 8.60$ ) from the healthy person's image (Fig. 4a) as a threshold. If the gray value of a pixel is within the range of the threshold, this pixel is classified as normal (colored black); otherwise, it is classified as abnormal (colored white). The binary-value image obtained is shown in Figure 5a. Observe that there are few damaged liver cells.

Now let us conduct normalized processing to the other 5 images. Mean $i$ be the mean gray level of the $i$-th image. Multiply a factor k $i=$ mean $1 /$ mean $i$ for the gray levels of the $i$-th normalized image. Then use the mean \pm standard deviation of the normalized image as a threshold. The binary-value images obtained are shown in Figure $5 b-f$. Observe that there are many regions with tooth-shaped white patterns or rectangle-shaped white patterns. They represent abnormal liver cells. Observe that the images in Figure 5c, e, and f are similar to the images shown as one of the candidates for modeling and interpreting HBV-infected patients' digital liver B-scan images (Fig. 2d-f); the images shown in Figure $5 b$ and d are similar to those shown in Figure $2 \mathrm{~g}$ and $\mathrm{h}$. This example suggests that the SR CNN may be used as a candidate for modeling and interpreting HBV-infected patients' digital liver B-scan images. 
Integrative

Medicine

Internationa

Fig. 4. Selected inset areas from the digital B-scan images shown in Figure 3. a Normal person. b Healthy HBV carrier. c-f Four chronic HBV patients.

Fig. 5. Selected inset areas from the binary digital B-scan images shown in Figure 4. a Normal person. b Healthy HBV carrier. c-f Four chronic HBV patients.
Wang et al.: Dynamic Analysis of Coupled Sawtooth and Rectangle Cellular Neural Networks with Application in HBV Patients' B-Scan Images
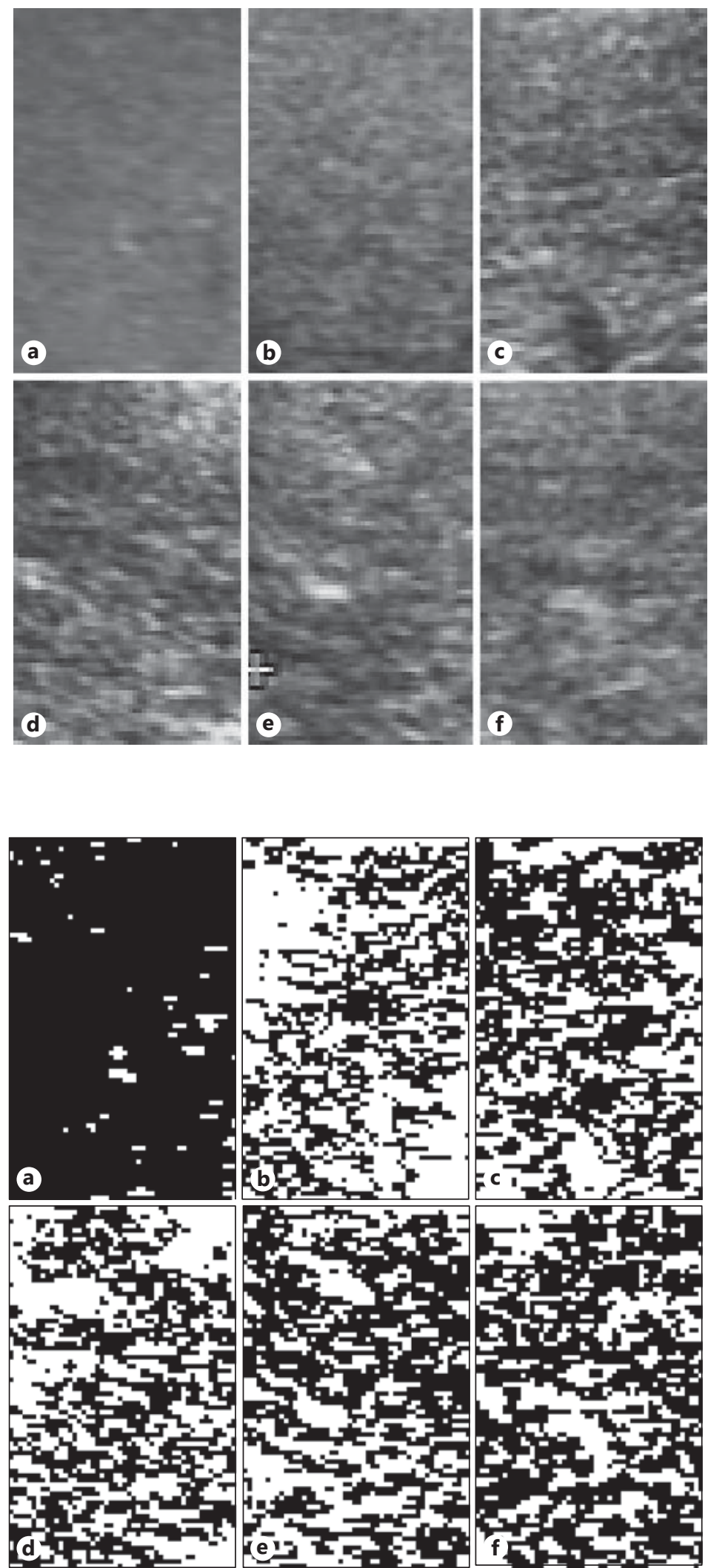


\section{Conclusions}

The SR CNN with the square cross template is able to generate output images in which sawtooth- and rectangle-shaped patterns coexist for any random-input grayscale pattern. These output images have characteristics appearing in some HBV-infected patients' digital liver B-scan images.

The dynamic behaviors of the SR CNN have not been studied mathematically since its introduction in 1995 [18]. This paper presented a mathematical dynamic analysis of the SR CNN and discussed its application for interpreting some HBV-infected patients' digital liver B-scan images. The results suggest that randomly replicated HBV via infected liver cells infects neighboring cells in a square cross way, generating sawtooth- and rectangle-like infection patterns.

In our paper, we introduced 6 definitions: inherent (final inherent) active cells (networks), inherent (final inherent) passive cells (networks), and inherent (final inherent) neutral cells (networks). These definitions describe fundamental characteristics and have a significant biological meaning.

We presented 6 theorems on the output images of the SR CNN. These theorems describe the dynamic behaviors of the SR CNN in a strict manner and answer why the SR CNN is able to generate such fantastic output images. The simulation examples confirm that the results of the theoretical analysis are efficient in their realization of numerical computer simulations.

In practice, it is difficult to confirm whether a cell is inherent (final inherent) active, inherent (final inherent) passive, or inherent (final inherent) neutral. However, these concepts suggest that the pixels of the output patterns of the SR CNN are classified into 3 kinds:

1. Active networks: they may represent a kind of specific substance or a specific pattern

in which sawtooth and rectangle shapes coexist, i.e., normal (uninfected or undamaged) cells.

2. Passive networks: they may represent another kind of specific substance or another

specific pattern in which sawtooth and rectangle shapes coexist, i.e., abnormal (infected or damaged) cells.

3. Neutral networks: they may represent interim substances or an interim pattern in which sawtooth and rectangle shapes coexist, i.e., interim (infecting or damaging) cells. Using a so-called threshold approach, we processed 6 people's digital liver B-scan images. The 6 binary images generated display patterns in which sawtooth and rectangle shapes coexist. The results imply that the SR CNN may be used as a liver infection model, and they provide a possible interpretation of HBV-infected patient's digital liver B-scan images.

There is the important issue to investigate the dynamics of more general CNNs with a coupled A template in order to more reasonably interpret HBV-infected patients' liver B-scan images. Research along this line is promising.

\section{Appendix}

PROOF OF THEOREM 1. If the stable output images of the SR CNN have a pattern like that shown in Figure 6b, according to local rule 1 and Figure 1, for the cell $C_{4,8}$ (it is a final active cell), there is a $t^{*}$ such that for $t \geq t^{*}$,

$$
\begin{aligned}
w_{4,8}(\infty) & =c\left(y_{4,7}(t)+y_{4,9}(t)+y_{3,8}(t)+y_{5,8}(t)\right) \\
& =c(1+1-1+1)=2 c \geq 1-a, \text { if } a<1, \\
w_{4,8}(\infty) & =c\left(y_{4,7}(t)+y_{4,9}(t)+y_{3,8}(t)+y_{5,8}(t)\right) \\
& =c(1+1-1+1)=2 c>0 \text { if } a=1 .
\end{aligned}
$$



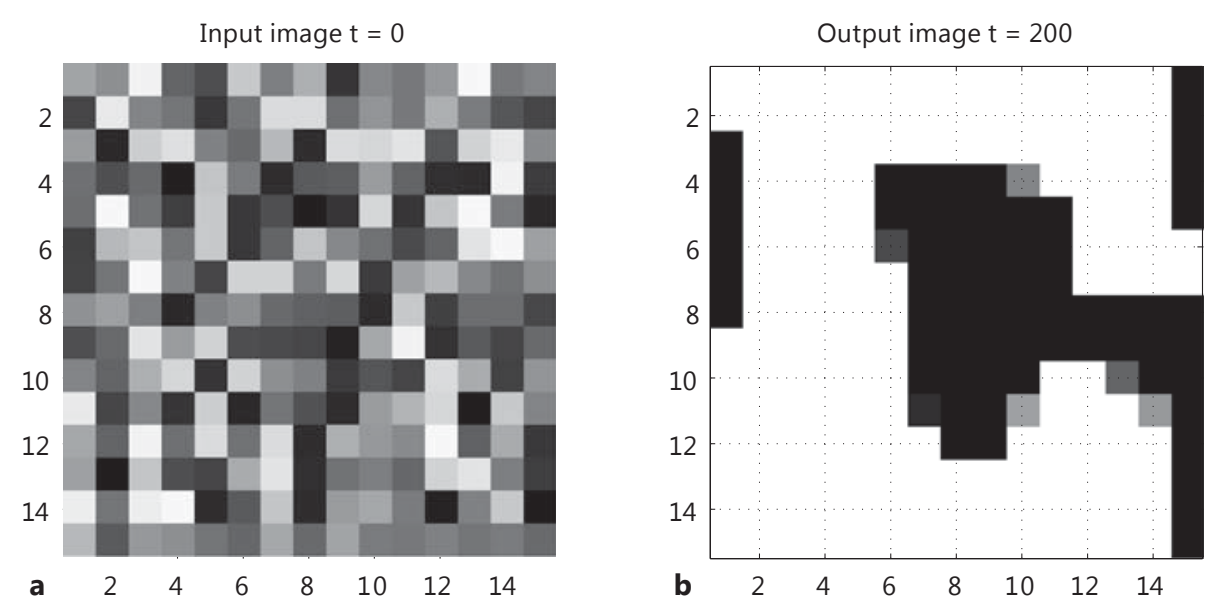

Fig. 6. Input pattern (a) and stable output pattern generated by the SR CNN (b).
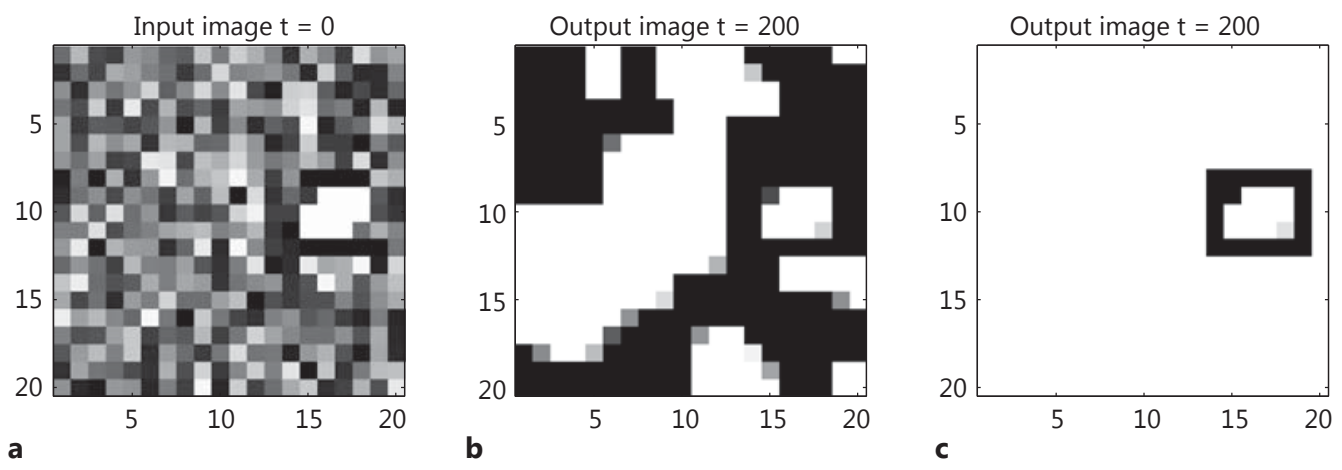

Fig. 7. a Input pattern. b Output pattern. c Selected unchanged sawtooth white island shown in a and b.

Hence Formulas (7), (8), and (9) must hold.

PROOF OF THEOREM 2. If there are at least 3 nearest fixed black neighbors on the nearest square cross lines, then

$$
w_{i, j}(t) \geq c(3-1)=2 c \geq 1-a, \forall t \geq 0 \text { if } a<1
$$

or

$w_{i, j}(t) \geq c(3-1)=2 c>0, \forall t \geq 0$ if $a=1$.

From Figure $1 y_{i, j}(t) \equiv 1$, it follows that $C_{i, j}$ is an inherent active cell.

PRoOf OF THEOREM 3. Similar to Theorem 2.

PROOF OF THEOREM 4. It can be concluded from Figure $1 \mathrm{a}$ and $\mathrm{b}$.

PROOF OF THEOREM 5.

(1) Assume that an isolated gray pixel at $(i, j)$ is surrounded by black pixels. Then $w_{i, j}=4 c$ $>1-a$ if $a<1$ or $w_{i, j}=4 c>0$ if $a=1$. In both cases, $y_{i, j}(\infty)=1$. This contradicts the assumption 


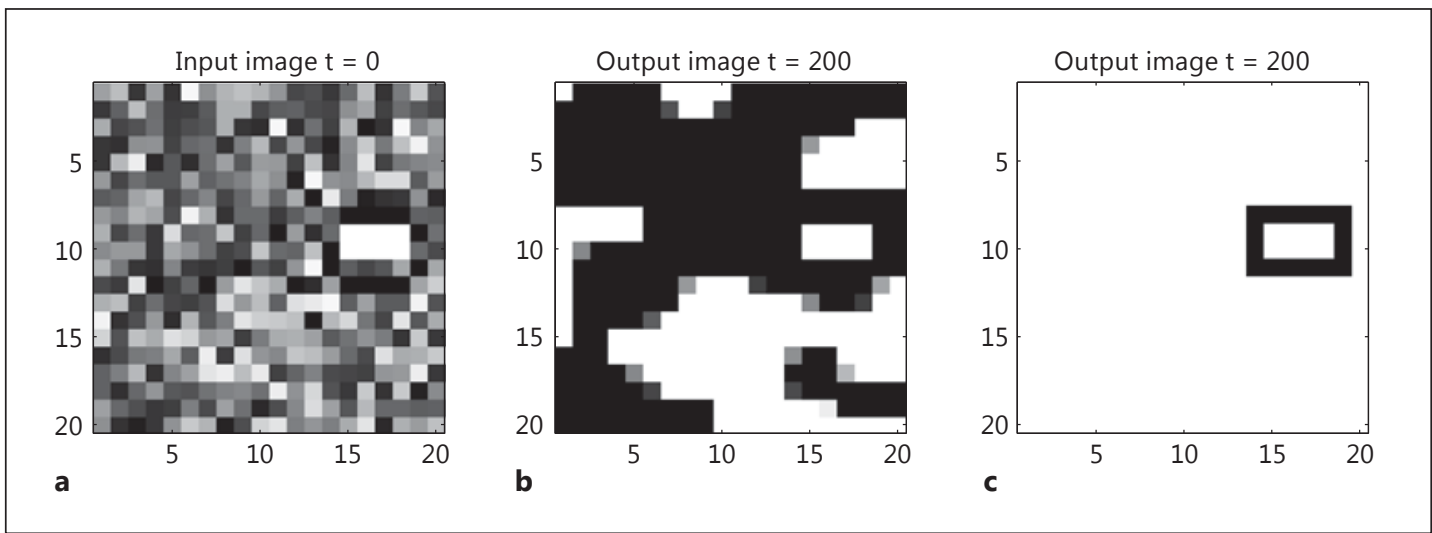

Fig. 8. a Input pattern. b Output pattern. c Selected unchanged rectangle white island shown in a and $\mathbf{b}$.

that $y_{i, j}(\infty)$ is gray. Similarly, we can show that if an isolated gray pixel at $(i, j)$ is surrounded by white pixels, then an isolated gray pixel at $(i, j)$ is surrounded by black pixels $y_{i, j}(\infty)=-1$.

(2) Assume that 2 isolated edge-connected gray pixels at $(i, j)$ and $(i, j+1)$ are surrounded by black pixels. Then $w_{i, j}>2 c \geq 1-a$ if $a<1$ or $w_{i, j}>2 c>0$ if $a=1$. In both cases, $y_{i, j}(\infty)=1$. This contradicts the assumption that $y_{i, j}(\infty)$ is gray. Similarly, we can show that if the 2 gray pixels are surrounded by white pixels, then an isolated gray pixels at $(i, j)$ are surrounded by black pixels $y_{i, j}(\infty)=-1$. In summary, this completes the proof.

PROOF OF THEOREM 6.

(1) The images in Figure 7a and $\mathrm{b}$ are the input image and the stable output image of an SR CNN, respectively. Observe that there is an unchanged sawtooth white island consisting of 10 cells in Figure 7a and b. Now, we select the sawtooth white island and its surrounding cells (as shown in Fig. 7c). It follows for every cell $C_{i, j}$ in the sawtooth white island that $w_{i, j}(0) \leq 0$. Consequently, $w_{i, j}(t) \leq 0$. This implies that $y_{i, j}(\infty)=-1$. Similarly, we can show that any sawtooth black island is unchanged. This completes the proof.

(2) The images in Figure 8a and b are the input image and the stable output image of an SR CNN, respectively. Observe that there is an unchanged rectangle white island consisting of 8 cells in Figure $8 a$ and $b$. Now, we select the rectangle white island and its surrounding cells (as shown in Fig. 8c). It follows for every cell $C_{i, j}$ in the rectangle white island that $w_{i, j}(0) \leq 0$. Consequently, $w_{i, j}(t) \leq 0$. This implies that $y_{i, j}(\infty)=-1$. Similarly, we can show that any rectangle black island is unchanged. This completes the proof.

\section{Acknowledgment}

L. Min would like to express his gratitude to Prof. Leon O. Chua for directing him to study the fascinating $\mathrm{CNN}$, and to anonymous reviewers for very valuable suggestions. This project is supported by the National Natural Science Foundation of China (grant No. 61074192). The authors are grateful to Dr. Shubiao Gao at Beijing University of Chinese Medicine for providing digital liver images. 
Wang et al.: Dynamic Analysis of Coupled Sawtooth and Rectangle Cellular Neural Networks with Application in HBV Patients' B-Scan Images

\section{References}

WHO: Hepatitis B. 2014. http://www.who.int/entity/mediacentre/factsheets/fs204/en/.

2 Chua LO, Yang L: Cellular neural networks: theory. IEEE Trans Circuits Syst 1988;35:1257-1272.

3 Chua LO, Yang L: Cellular neural networks: applications. IEEE Trans Circuits Syst 1988;35:1273-1290.

4 Chua LO: Passivity and complexity. IEEE Trans Circuits Syst 1999;46:72-82.

5 Chua LO: CNN: a version of complexity. Int J Bifurcat Chaos 1997;7:2219-2425.

6 Chua LO: Special issue on nonlinear waves, patterns and spatio-temproal chaos in dynamic arrays (guest editorial). IEEE Trans Circuits Syst 1995;42.

7 Orzo L, Vidnyanszky Z, Hamori J, Roska T: CNN model of the feature linked synchronized activities in the visual thalamo-cortical system; in Workshop on Cellular Neural Networks and Their Application (CNNA-96), Seville, Spain, 1988, pp 1257-1272.

8 Weblin F, Jacobs A, Teeters J: The computational eye. IEEE Spectrum, May, 1996, pp 30-37.

$\checkmark 9$ Dogaru R, Chua LO: Universal CNN cells. Int J Bifurcat Chaos 1999;9:1-48.

10 Chua LO, Roska T: Cellular Neural Networks and Visual Computing. Cambridge, Cambridge University Press, 2002.

11 Arena P, Basile A, Fortuna L, Frasca M: CNN wave based computation for robot navigation; in International Symposium on Circuit and Systems (ISCAS 2004), 2004, vol 5, pp 500-503.

$\$ 12$ Liu J, Min L: Robust designs for gray-scale global connectivity detection CNN templates. Int J Bifurcat Chaos 2007; 17:2827-2838.

13 Li G, Min L, Zang H: Color edge detections based on cellular neural network. Int J Bifurcat Chaos 2008;18: 1231-1242.

14 Zang H, Min L: Generalized synchronization theorems for a kind of neural network with application in data encryption; in Proceedings of the 3rd IEEE Conference on Industrial Electronics and Applications, Singapore, 2008, pp 948-953.

15 Babatunde $\mathrm{H}$, Folorunso O, Akinwale A: A cellular neural network-based model for edge detection.J Inf Comput Sci 2010;5:003-010.

-16 Liu J, Min L: Robust design of bipolar wave cellular neural network with applications. Int J Model Ident Contr 2010;11:99-106.

17 Li J, Bai F, Di X: New color image encryption algorithm based on compound chaos mapping and hyperchaotic cellular neural network. J Electron Imaging 2013;22:013036.

18 Hanggi M, Moschytz GS: Genetic optimization of cellular neural networks; in Proceedings of the IEEE International Conference on Evolutionary Computation, Anchorage, 1988, pp 381-386.

19 Thiran P, Crounse KR, Chua LO, Hasler M: Pattern formation properties of autonomous cellular neural networks. IEEE Trans Circuits Syst 1995;42:757-774. 